\title{
Collagen peptide provides Streptomyces coelicolor CGMCC 4.7172 with abundant precursors for enhancing undecylprodigiosin production
}

\author{
Xia Li ${ }^{1,2}$, Meifeng Li ${ }^{1}$, Junling Guo ${ }^{1}$, Xian Liu ${ }^{1,3}$, Xuepin Liao ${ }^{1,2,3^{*}}$ (i) and Bi Shi ${ }^{1,2,3}$
}

\begin{abstract}
Effective and ecofriendly converting biomass to chemicals is important for sustainable engineering based on the foreseeable shortage of fossil resources. Undecylprodigiosin (UP) is a promising antibiotic, but the direct feeding of pure precursor amino acids makes it costly for large-scale production. Here, collagen peptide (CP), a renewable animal-derived biomass contains abundant precursor amino acids of UP. CP can act as carbon and nitrogen source for the growth of Streptomyces coelicolor CGMCC 4.7172. The plant biomasses including soybean meal, wheat bran, and malt extract were unsuitable for UP prodution. However, $365.40 \mu \mathrm{g} / \mathrm{L}$ UP was detected after $24 \mathrm{~h}$ in the media containing CP, and its highest concentration reached $1198.01 \mu \mathrm{g} / \mathrm{L}$. UP was also detected in the media containing meat hydrolysates of domestic animals, but its initial production time was delayed, and final concentration was lower than that in the medium containing CP only. Compared the fermentation performances of CP and other proteins, CP has a special superiority for UP production. These results revealed that UP biosynthesis may be dependent on amino acid availability of substrates and CP is beneficial for UP production because of its specific amino acid composition.
\end{abstract}

Keywords: Biomass, Collagen peptide, Fermentation, Streptomyces coelicolor, Antibiotic, Undecylprodigiosin

\section{Introduction}

Biomass has attracted significant attention as a sustainable resource because of increasing environmental concerns and decreasing fossil resources [1,2]. Biomass can be used to produce important chemicals, such as intermediates, bioenergy, and bioactive molecules related to various aspects of human beings [3, 4]. Nowadays, plant biomass has been extensively exploited, whereas animal biomass is largely underutilized despite its enormous potential due to the limitations of low-value conversion approaches [5]. Therefore, developing an effective and

\footnotetext{
*Correspondence: xpliao@scu.edu.cn

'Department of Biomass Science and Engineering, Sichuan University, 610065 Chengdu, People's Republic of China

${ }^{2}$ National Engineering Research Center of Clean Technology in Leather Industry, Sichuan University, 610065 Chengdu, People's Republic of China Full list of author information is available at the end of the article
}

ecofriendly strategy for converting animal biomass to high-value products is particularly important.

Antibiotic is an important pharmaceutical used for humans and domestic animals. The majority of clinical antibiotics of natural origin are produced by Streptomyces species [6]. As a model organism, Streptomyces coelicolor can produce antibiotics mainly including redpigmented undecylprodigiosin (UP), blue-pigmented Type II polyketide actinordin, and calcium-dependent lipopeptide antibiotic [7]. Especially, great attention was focused on the UP owing to its immunosuppressive and anticancer properties, in addition to antimicrobial activities $[8,9]$. Ho et al. [10] have reported that UP can selectively induce apoptosis in human breast carcinoma cells independent of p53. Thus, UP is a promising antibiotic in the pharmaceuticals industry. Current strategies 
to achieve high UP yield include exploitation of new strains by efficient isolation, construction of industrial producers by metabolic engineering, and supplementation of special precursors by direct feeding [11-14]. In fact, exogenous feeding with special precursors is a pioneering approach to improve antibiotic yield during fermentation.

UP is a member of the family of prodiginines, which are structurally characterized by a common pyrrolylpyrromethene skeleton [15]. Precursors of UP mainly includes acetyl-CoA, malonyl-CoA, proline (Pro), glycine (Gly), and serine (Ser) [16]. Acetyl-CoA is generally derived from the degradation pathway of common sugars, lipids and proteins [17]. Moreover, malonyl-CoA can be obtained by the bioconversion of acetyl-CoA with acetyl-CoA carboxylase [18]. Considering the limitation of common substrates, precursor amino acids (AAs), including Gly, Pro and Ser are generally used in exogenous feeding as the nitrogen source for UP biosynthesis. However, pure free AAs are costly for large-scale fermentation. Therefore, a novel resource of precursors should be developed to achieve the cost-effective production of UP.

Collagen $\mathrm{I}$ is an abundant renewable animal biomass, which can be easily extracted from the skins, bones, tendons and cartilage of domestic animals [19-21].The collagen triple- helix consists of a repeating $(\mathrm{Gly}-\mathrm{X}-\mathrm{Y})_{\mathrm{n}}$ sequence, and the AAs in the $\mathrm{X}$ and $\mathrm{Y}$ positions of collagen are generally Pro and hydroxyproline (Hyp), respectively [19, 22, 23]. The fact illustrated that the Gly-Pro-Hyp is the most common triplet in collagen. Collagen peptide $(\mathrm{CP})$, prepared by the hydrolyzation of collagen I, is easier utilized by microorganisms than collagen [24, 25]. Moreover, CP contains abundant Gly and Pro as UP precursors, based on its unique composition of AAs. In particular, Thomas et al. [26] proved that the pyrrolidine ring of Pro can be incorporated into a pyrrole of UP within its biosynthetic pathways. Besides, Ser is also included in $\mathrm{CP}$, which is also a necessary AA for UP biosynthesis. Accordingly, the AAs composition of $\mathrm{CP}$ makes it an ideal substrate for UP production.

In the present study, the effects of different $\mathrm{CP}$ concentrations on the growth profile of S. coelicolor CGMCC 4.7172 (China General Microbiological Culture Collection Center) were investigated. Besides, the UP producing abilities using $\mathrm{CP}$ and these plant biomasses were compared. Meanwhile, the influence of $\mathrm{CP}$ on the UP production in the media containing soybean meal $(\mathrm{SM})$ and wheat bran (WB) were analyzed, respectively. For comparison, the investigation of antibiotic producing abilities involved in meat hydrolysates of rabbit, beef, mutton, chicken, and duck were also conducted. The fermentation performances using bovine serum albumin (BSA), casein, and keratin as substrates were also investigated to understand the effect of CP.

\section{Materials and methods}

\subsection{Microorganism and raw materials}

The Streptomyces coelicolor CGMCC 4.7172 utilized in this study was obtained from the China General Microbiological Culture Collection Center (CGMCC). Collagen peptide $(\mathrm{CP})$ was prepared though the enzymatic degradation of collagen I following the method developed in our previous work [25], and its weight-average molecular weight was $2900 \mathrm{Da}$. Soybean meal (SM), and wheat bran (WB) were friendly offered by Mianyang Habio Bioengineering Co., Ltd. Soybean powder (SP), and the meat of rabbit, beef, mutton, chicken, and duck were purchased from a local vegetable market. Besides, keratin (from wool) was provided by Tokyo Chemical Industry Co., Ltd. Yeast extract and tryptone were obtained from Oxoid (Cambridge, U.K.). Bovine serum albumin (BSA) and other chemicals were provided by Aladdin (Shanghai, China). The undecylprodigiosin (UP) standard for quantitative analysis was purchased from Abcam (Hong Kong, China).

\subsection{Media and cultivation conditions}

S. coelicolor CGMCC 4.7172 was routinely maintained on glucose-yeast-malt agar containing $(w / v) 0.4 \%$ glucose, $0.4 \%$ yeast extract, $1.0 \%$ malt extract (ME), $0.2 \%$ $\mathrm{CaCO}_{3}$, and $1.5-2 \%$ agar powder. Moreover, the seed medium was composed of $(w / v) 3.0 \%$ yeast extract, $3.0 \%$ tryptone, $0.5 \%$ glucose, $0.4 \%$ maltose, $0.2 \%$ $\mathrm{MgSO}_{4} \cdot 7 \mathrm{H}_{2} \mathrm{O}$. The spore suspension of S. coelicolor was first inoculated into seed culture medium at a final spore count of $2 \times 10^{6}$ spores $/ \mathrm{mL}$, and the strain was cultivated for 2 days (d) in an orbital shaker at $30{ }^{\circ} \mathrm{C}$ and $200 \mathrm{rpm}$, which served as an inoculum of the different fermentation media. The cells were inoculated into $250 \mathrm{~mL}$ flasks containing $120 \mathrm{~mL}$ of fermentation media, and cultured at $30{ }^{\circ} \mathrm{C}$ with shaking at $200 \mathrm{rpm}$ for different time. Moreover, all initial $\mathrm{pH}$ values of these fermentation media were adjusted to 7.0-7.2 and followed its natural course.

\subsubsection{Effect of different CP concentrations in ISP-2 media} International Streptomyces Project medium No.2 (ISP-2) for fermentation was composed of $(w / v) 0.4 \%$ glucose, $0.4 \%$ yeast extract, and $1.0 \%$ ME. Moreover, different concentrations of CP were separately added into ISP-2 medium to form different fermentation media. The cultivated cells were directly inoculated at a concentration of $5 \%(v / v)$ into these media. 


\subsubsection{Comparison of $\mathrm{CP}$ with other biomasses}

The minimal medium (MM), including ( $w / v) \quad 0.05 \%$ $\mathrm{KH}_{2} \mathrm{PO}_{4}, 0.15 \% \mathrm{Na}_{2} \mathrm{HPO}_{4}, 0.10 \% \mathrm{NaCl}$, and $0.02 \%$ $\mathrm{MgSO}_{4} \cdot 7 \mathrm{H}_{2} \mathrm{O}$, was used as basal culture media. Furthermore, $\mathrm{CP}$ and other various substrates were added into MM media to form different culture media. The cultivated cells in seed media were collected, washed, and resuspended in equal volume of sterile $0.85 \%(w / v) \mathrm{NaCl}$. The prepared cells were inoculated at a concentration of $5 \%(v / v)$ in different culture media.

\subsection{Analytical methods}

An aliquot of S. coelicolor CGMCC 4.7172 culture was harvested by centrifugation, and then suspended in $0.5 \mathrm{~mol} / \mathrm{L} \mathrm{HCl}$ methanol for UP extraction. The UP concentration was analyzed by high-performance liquid chromatography (HPLC, Agilent 1260 Infinity, Agilent Technologies, SantaClara, US-CA) with a Zorbax Eclipse Plus C18 column $(250 \times 4.6 \mathrm{~mm}$, i.d.; $5 \mu \mathrm{m})$ and a diodearray detector. The column temperature was $30{ }^{\circ} \mathrm{C}$ with a constant flow rate at $0.6 \mathrm{~mL} / \mathrm{min}$ and an absorbance at $530 \mathrm{~nm}$. The mobile phase was a gradient prepared from methanol (A) and $0.1 \%$ formic acid in water (B) according to the following program: $0-10 \mathrm{~min}, 5-100 \% \mathrm{~A} ; 10$ $18 \mathrm{~min}, 100 \% \mathrm{~A} ; 18-20 \mathrm{~min}, 100-5 \% \mathrm{~A} ; 20-25 \mathrm{~min}$, $5 \% \mathrm{~A}$.

The carbon-to-nitrogen $(\mathrm{C} / \mathrm{N})$ ratio of the substrates were calculated by measuring their content of $\mathrm{C}$ and $\mathrm{N}$ using a Flash EA $1112 \mathrm{NC}$ analyzer (Thermo Fisher Scientific Inc., Waltham, MA, USA) [27, 28]. Besides, the amino acids (AAs) compositions of these substrates were determined by an A300 fully automatic AA analyzer (Membrapure, Berlin, Germany) [29]. These substrates were hydrolyzed with $6 \mathrm{~mol} / \mathrm{L} \mathrm{HCl}$ at $120{ }^{\circ} \mathrm{C}$ for $24 \mathrm{~h}$, and the hydrolysates were used to analyze the contents of the corresponding AAs [30]. In addition, the cell growth was characterized as dry cell weight (DCW). The cell pellets in an aliquot culture were collected, washed, and then dried at $55{ }^{\circ} \mathrm{C}$, until the weight remained stable [31]. Meanwhile, the contents of total organic carbon (TOC) and total chemically bound nitrogen (TNb) of the culture supernatant during fermentation were measured using a $\mathrm{C}$ and $\mathrm{N}$ analyzer (Vario TOC, Elementar, Hanau, Germany) [32, 33].

\subsection{Effect of CP on the growth profile of the S. coelicolor}

\subsubsection{Effect of different $C P$ concentrations}

ISP-2 media were supplemented with $0.1 \%, 1 \%, 2 \%$, $3 \%, 4 \%$, and $5 \%(w / v) \mathrm{CP}$ and labeled as ISP-2_0.1CP, ISP-2_1CP, ISP-2_2CP, ISP-2_3CP, ISP-2_4CP and ISP2_5CP media, respectively. The cultivated cells were directly inoculated to these media to explore the influence of CP concentrations on the growth of $S$. coelicolor. The
$\mathrm{pH}$ value and $\mathrm{DCW}$ were determined during the fermentation period of $13 \mathrm{~d}$.

\subsubsection{Growth profile of S. coelicolor when CP acted as the sole source of carbon and nitrogen}

MM medium, containing only inorganic salt without any source of carbon and nitrogen, was served as control. According to the pre-experimental results (Supplementary Note 1, and Fig. S1a and b), the concentration of $\mathrm{CP}$ was determined as $2 \%(w / v)$ to investigate the fermentation performances of $S$. coelicolor. Thus, the prepared cells, almost without the components of seed media, were inoculated into the MM and 2CP (MM media containing $2 \%(w / v) \mathrm{CP})$ media to investigate the growth behavior of the $S$. coelicolor, when CP was used as the sole source of carbon and nitrogen. The $\mathrm{pH}$ values and $\mathrm{DCW}$ were tested during the culture period.

\subsection{Comparison of UP biosynthesis performance between $\mathrm{CP}$ and plant biomass}

\subsubsection{UP producing abilities of $C P$ and plant biomass}

MM media were separately supplemented with $(w / v) 2 \%$ $\mathrm{SM}, 2 \% \mathrm{SP}, 2 \% \mathrm{WB}$, and $2 \% \mathrm{ME}$, and designed as $2 \mathrm{SM}$, 2SP, $2 \mathrm{WB}$ and $2 \mathrm{ME}$ media, which were used to compare the UP producing abilities between $\mathrm{CP}$ and plant biomass. The UP concentration was measured during the fermentation period of $13 \mathrm{~d}$. Moreover, the CP concentration added in MM media was increased to $4 \%(w / v)$, which was designed as $4 \mathrm{CP}$ media. Meanwhile, the added concentration of plant biomass was also doubled, and the fermentation period was prolonged to $20 \mathrm{~d}$ for gaining insight into the difference of UP producing ability between $\mathrm{CP}$ and the four plant biomasses. The MM media containing $(w / v) 4 \% \mathrm{SM}, 4 \% \mathrm{SP}, 4 \% \mathrm{WB}$, and $4 \% \mathrm{ME}$ were labeled as $4 \mathrm{SM}, 4 \mathrm{SP}, 4 \mathrm{WB}$, and $4 \mathrm{ME}$ media, respectively. In addition, the Pro, Gly and Ser contents of the biomass resources, including $\mathrm{CP}, \mathrm{SM}$, $\mathrm{SP}, \mathrm{WB}$, and ME, were analyzed.

\subsubsection{Effect of CP on UP fermentation}

MM media supplemented with $(w / v) 2 \%$ SM and $2 \%$ $\mathrm{CP}$ was deemed as 2SM_2CP media. Similarly, MM media containing $(w / v) 2 \% \mathrm{WB}$ and $2 \% \mathrm{CP}$ was denoted as $2 \mathrm{WB} \_2 \mathrm{CP}$ media. The $\mathrm{pH}$ value and UP concentration were measured to evaluate the effect of $\mathrm{CP}$ on fermentation by the strain S. coelicolor.

\subsection{Fermentation performance of meat hydrolysates by $S$. coelicolor CGMCC 4.7172}

Meat hydrolysates were prepared by the enzyme hydrolysis of rabbit, beef, mutton, chicken, and duck as previously described [25]. The $2 \%(w / v)$ meat hydrolysate of rabbit, beef, mutton, chicken, and duck were contained in $\mathrm{MM}$ media, respectively. The $\mathrm{C} / \mathrm{N}$ ratios of 
these substrates were analyzed. Meanwhile, the contents of hydroxyproline (Hyp) included in $\mathrm{CP}$ and these animal biomasses were determined. In addition, the $\mathrm{pH}$ value and UP concentration were measured during the fermentation period of $20 \mathrm{~d}$.

\subsection{UP producing ability of animal protein}

MM media including $(w / v) 2 \%$ BSA, $2 \%$ casein, and $2 \%$ keratin were used to study the antibiotics producing ability of animal protein. The contents of Pro, Gly, and Ser and the $\mathrm{C} / \mathrm{N}$ ratio of these substrates were analyzed. In addition, the $\mathrm{pH}$ value and UP concentration were measured during the fermentation period of $20 \mathrm{~d}$.

\subsection{Statistical analysis}

Data were subjected to statistical analysis performed by SPSS (version 25.0 for windows, SPSS Inc., CO, USA) and graphs were created in Origin Pro 2017 (Origin Lab Corporation, Northampton, MA, USA). The results were expressed as mean \pm standard deviation (SD). One-way ANOVA with Tukey's HSD analysis were used to determine significance, and the level of significance was $\mathrm{p}<$ 0.05 .

\section{Results and discussion}

3.1 Effect of CP on the growth profile of the S. coelicolor

The different concentrations of $\mathrm{CP}$ were added into ISP-2 media to investigate the effect of CP addition on the growth of S. coelicolor CGMCC 4.7172. Actually, ISP-2 and YEME media are the common media for cultivating S. coelicolor [34-36]. It was found that the $S$. coelicolor in ISP-2 media had higher UP concentration but lower DCW than those in YEME media (Supplementary Note 1, and Fig. S1a and b). Hence, ISP-2 medium was used as a probe medium for further investigation of growth.

In general, the DCW of the $S$. coelicolor increased with the addition of CP (Fig. 1a). The highest DCW was reached at $5 \mathrm{~d}$ in ISP-2 media with $3.17 \mathrm{~g} / \mathrm{L}$, while the DCW increased to 4.40, 8.02, and $15.90 \mathrm{~g} /$ $\mathrm{L}$ in ISP-2_1CP, ISP-2_3CP, and ISP-2_5CP media at 3 d, respectively. Besides, the second growth of $S$. coelicolor was observed when more than $1 \%(w / v)$ $\mathrm{CP}$ was added, and the highest DCW was obtained in ISP-2_5CP at $13 \mathrm{~d}$ among these media. These results suggest that addition of $\mathrm{CP}$ can promote the growth of $S$. coelicolor. Moreover, $\mathrm{pH}$ is a comprehensive index reflecting the metabolic activity of microorganism [37]. At the early fermentation stage,
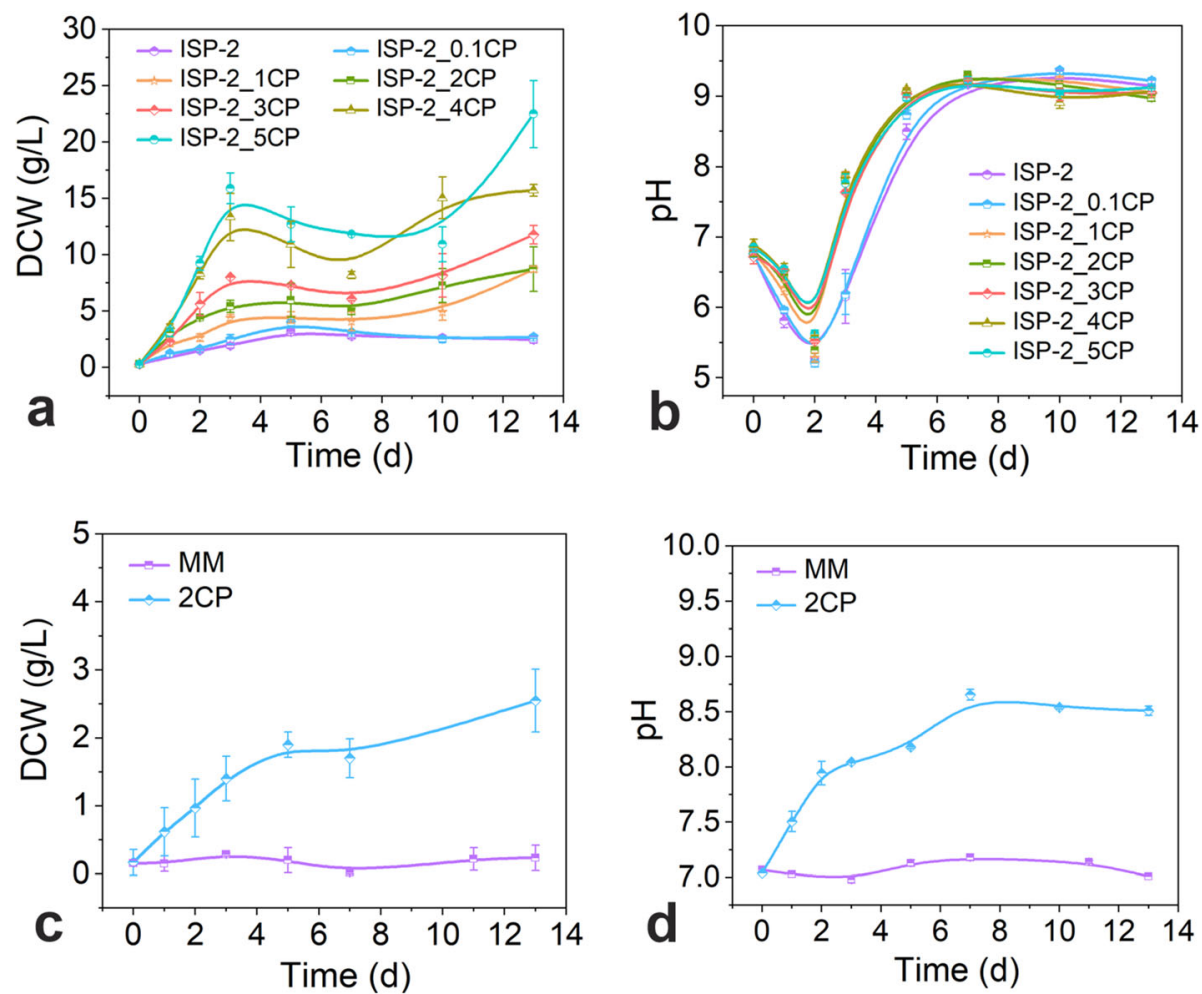

Fig. 1 Influence of CP on the growth behavior of the strain S. coelicolor CGMCC 4.7172. a and $\mathbf{b}$ Time course of DCW and pH values in the ISP-2, ISP-2_0.1CP, ISP-2_1CP, ISP-2_2CP, ISP-2_3CP, ISP-2_4CP and ISP-2_5CP media, respectively. $\mathbf{c}$ and $\mathbf{d}$ The change of DCW and pH values in the $\mathrm{MM}$ and $2 \mathrm{CP}$ media during the culture process of $13 \mathrm{~d}$, respectively 

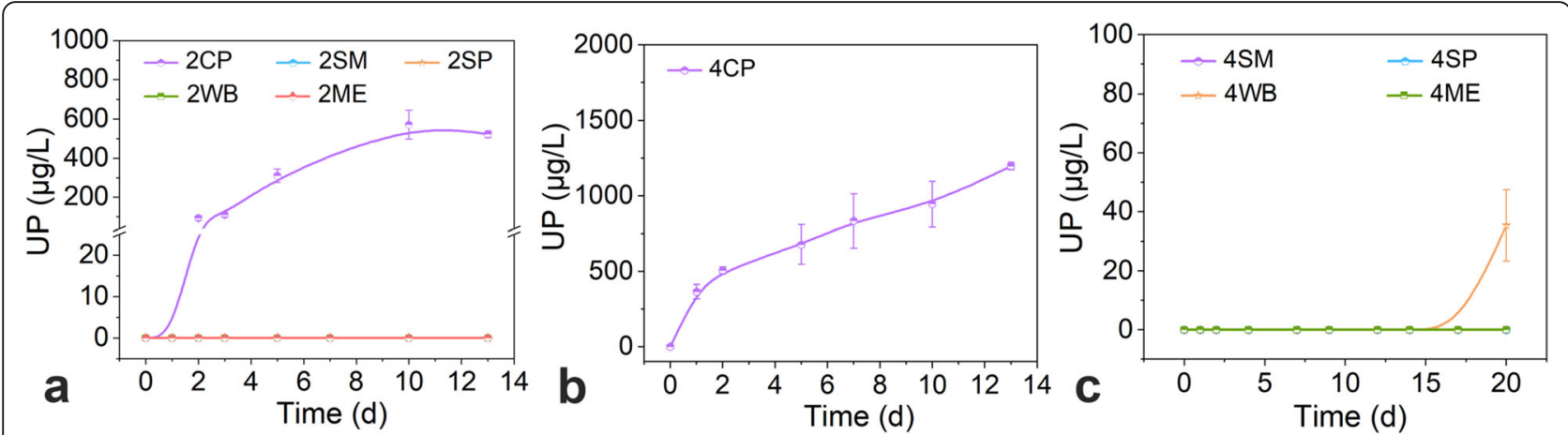

Fig. 2 Comparison of UP producing ability between CP and plant biomass by S. coelicolor CGMCC 4.7172. a The UP concentrations in the 2CP, 2SM, 2SP, 2WB and 2ME media, respectively, during the fermentation process of $13 \mathrm{~d}$. $\mathbf{b}$ The UP concentrations in the MM media supplemented with $4 \%(W / V)$ CP (4CP media). c The UP yields in 4SM, 4SP, 4WB, and 4ME media, respectively, during the fermentation period of $20 \mathrm{~d}$

the $\mathrm{pH}$ values were elevated when $\mathrm{CP}$ was added (Fig. 1b), which indicated that the existence of $\mathrm{CP}$ can change the metabolism of S. coelicolor, thereby increasing $\mathrm{pH}$ values.

Furthermore, the TOC and TNb of the fermentation broth increased, whereas the TOC/TNb declined with increasing $\mathrm{CP}$ concentration (Fig. S2), because $\mathrm{CP}$ also belongs to protein-based biomass. The strain $S$. coelicolor showed poor growth in MM media, which only contained inorganic salts without any carbon and nitrogen sources (Fig. 1c). However, when $2 \%(w / v)$ CP was added into MM media, the S. coelicolor exhibited normal growth during the culture

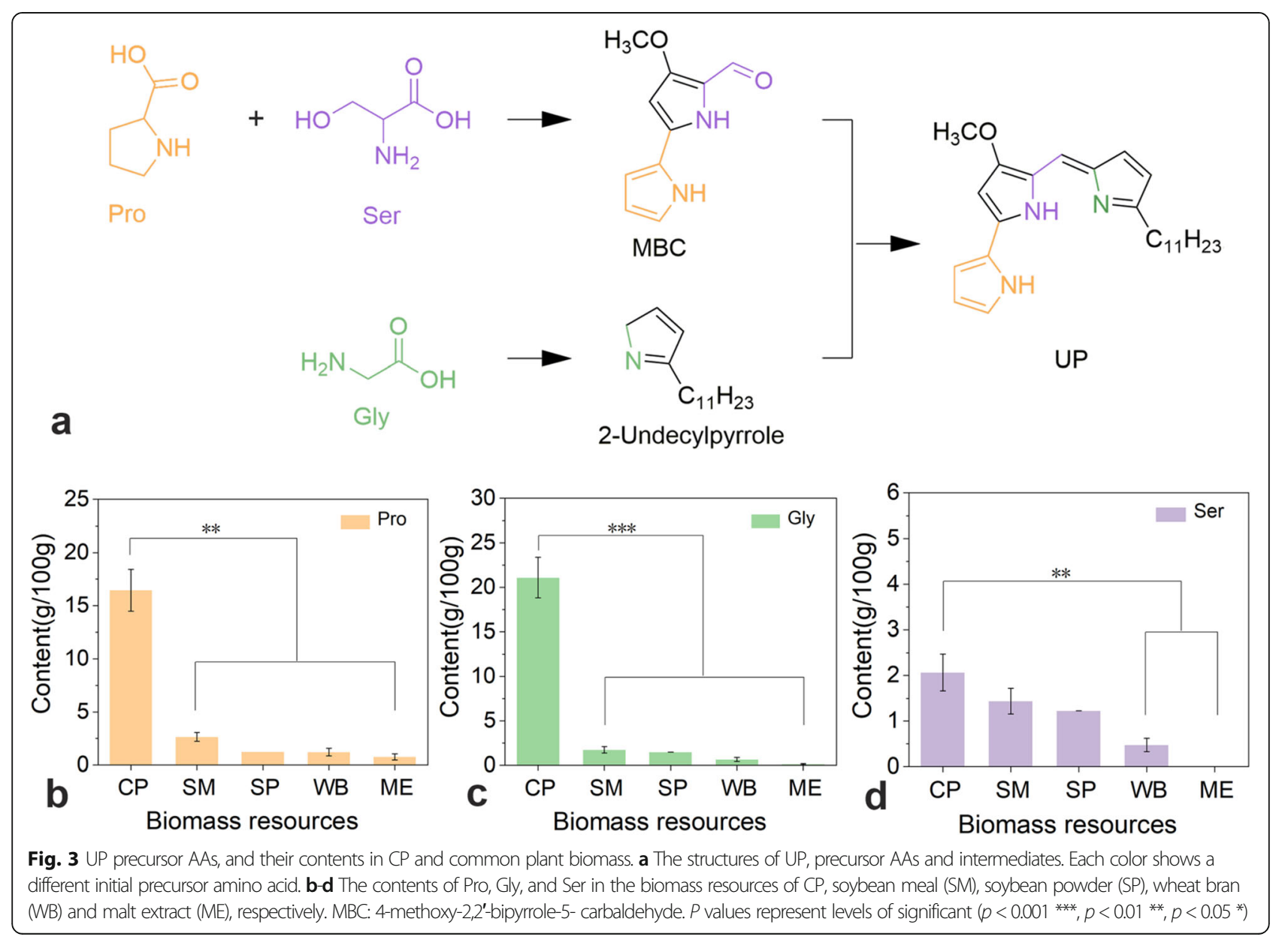



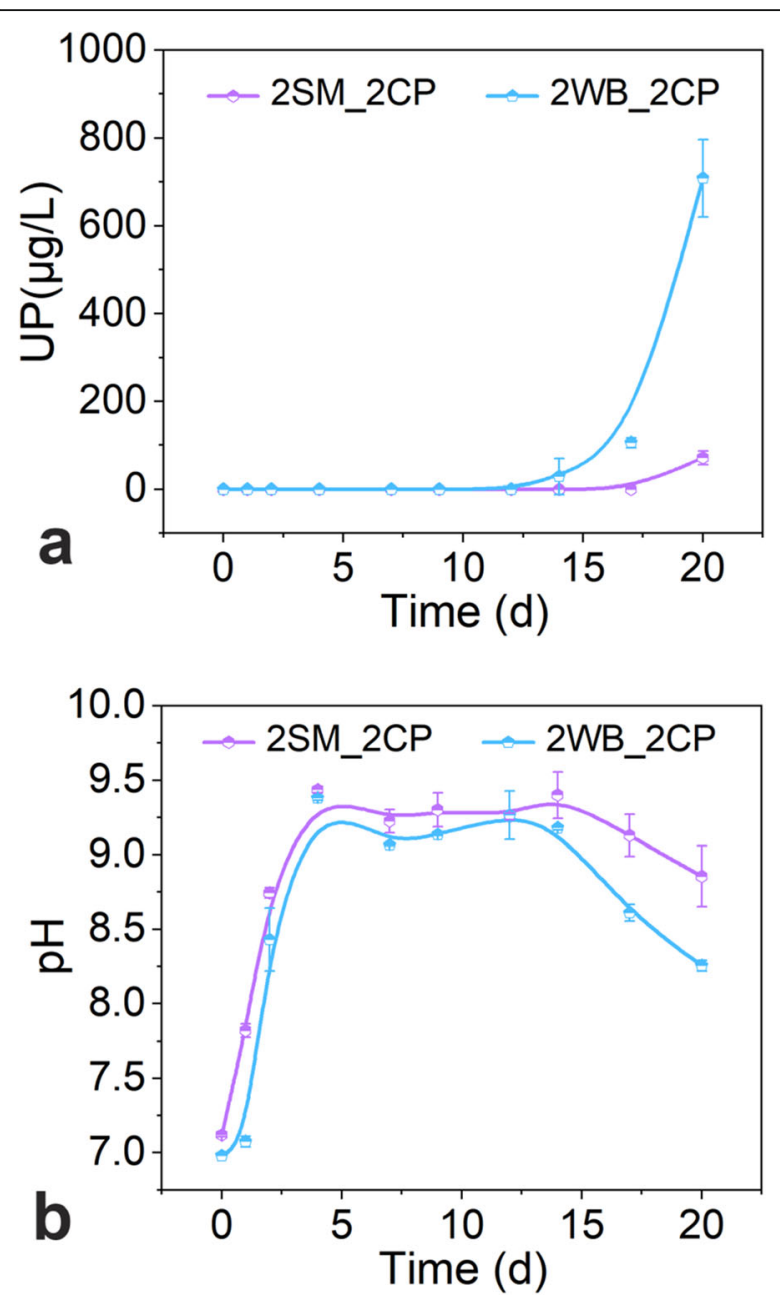

Fig. 4 Effect of CP on fermentation performance of S. coelicolor CGMC C 4.7172. $\mathbf{a}$ and $\mathbf{b}$ The variation of UP concentrations and $\mathrm{pH}$ values in 2SM_2CP and 2WB_2CP media, respectively, during the fermentation process of $20 \mathrm{~d}$

process. The results suggest that $\mathrm{CP}$ can be used as a carbon and nitrogen source for the growth of S. coelicolor. In addition, the $\mathrm{pH}$ values in MM media were almost unchanged throughout the fermentation period of $13 \mathrm{~d}$ (Fig. 1d). However, when $\mathrm{CP}$ was added into MM media, the $\mathrm{pH}$ values increased from the initial $\mathrm{pH} 7.04$ to the peak of 8.66 at $7 \mathrm{~d}$, which further demonstrated that CP can be utilized by the $S$. coelicolor.

\subsection{Comparison of UP biosynthesis performance between $\mathrm{CP}$ and plant biomass}

\subsubsection{UP producing abilities of CP and plant biomass}

Zang et al. [38] reported that the $\mathrm{C} / \mathrm{N}$ ratio of the media is critical for Serratia marcescens N10612 to produce prodigiosin isoform pigment. These plant biomasses with different $\mathrm{C} / \mathrm{N}$ ratios (Fig. S3) were separately added into MM media for the comparison with $\mathrm{CP}$ on antibiotic production. Virtually, SM, SP, WB, and ME are prevalent plant biomasses used in the fermentation industry. Surprisingly, UP was not detected in the 2SM, 2SP, $2 \mathrm{WB}$, and $2 \mathrm{ME}$ media during the whole fermentation period of $13 \mathrm{~d}$ (Fig. 2a). However, the UP concentration in the MM media with $2 \%(w / v) \mathrm{CP}$ was $93.06 \mu \mathrm{g} / \mathrm{L}$ at $2 \mathrm{~d}$, and then it increased to $571.94 \mu \mathrm{g} / \mathrm{L}$ after $10 \mathrm{~d}$. These results indicated that $\mathrm{CP}$ can be both used as carbon and nitrogen sources to produce UP.

Intriguingly, when the $\mathrm{CP}$ concentration increased from 2 to $4 \%(w / v)$, the UP was detected after $24 \mathrm{~h}$ with $365.40 \mu \mathrm{g} / \mathrm{L}$, and it reached the highest concentration at $13 \mathrm{~d}$ with $1198.01 \mu \mathrm{g} / \mathrm{L}$ (Fig. 2b). Moreover, the concentrations of plant biomasses also increased to $4 \%(w / v)$ for investigating the antibiotic producing abilities of $S$. coelicolor CGMCC 4.7172 in 4SM, 4SP, 4WB and 4ME media. It was found that only a small amount of UP was produced in $4 \mathrm{WB}$ media, and no UP was detected in other media, even though the fermentation time was prolonged to $20 \mathrm{~d}$ (Fig. 2c). These results suggested that SM, SP, WB and $\mathrm{ME}$ are not suitable for the strain to produce UP, while CP can enhance UP production.

It was reported that the biosynthesis of antibiotics in S. coelilcolor is greatly affected by precursor supply [39]. Stankovic et al. [11] have reported that the cell dry weight and UP concentration were increased by 1.46 and 1.49 folds, respectively, when MSY media was supplemented with $(w / v) 0.1 \%$ Pro, $0.1 \%$ Gly and $0.1 \%$ Ser. Wei et al.[40] have reported that UP concentration increased to 2.90 folds when $0.5 \%$ $(w / v)$ Pro was added into LB media by Serratia marcescens Simon Swift-1 (SS-1), suggesting that the addition of precursor amino acids can enhance UP production. UP is a tripyrrole antibiotic, which is condensed by two intermediates, 2-undecylpyrrole and 4-methoxy-2,2'-bipyrrole-5-carbaldehyde (MBC) [41] (Fig. 3a). Basically, the $\mathrm{N}$ contained in UP tripyrrole structure is obtained from Pro, Gly, and Ser, respectively [16, 42]. Hence, Pro, Gly, and Ser are essential precursor AAs for UP biosynthesis.

Interestingly, the contents of Pro, Gly, and Ser in CP are the highest among these biomass resources, which are roughly $16.45,21.10$, and $2.06 \mathrm{~g} / 100 \mathrm{~g} \mathrm{CP}$, respectively (Fig. 3b-d). The contents of Pro in ME, Gly in SM, and Ser in WB are only approximately $1 / 21,1 / 12$, and $1 /$ 4 folds those in $\mathrm{CP}$, respectively. Thus, a possible explanation for the stimulation of UP production could be the increased precursor availability due to $\mathrm{CP}$ supplementation. 


\subsubsection{Effect of CP on UP fermentation}

UP yield increased when SM and WB in $4 \mathrm{SM}$ and $4 \mathrm{WB}$ media were partly replaced by CP (Fig. $2 \mathrm{c}$ and $4 \mathrm{a}$ ). Surprisingly, the UP concentration increased from $35.37 \mu \mathrm{g} / \mathrm{L}$ in $4 \mathrm{WB}$ media to $708.60 \mu \mathrm{g} / \mathrm{L}$ in $2 \mathrm{WB} \_2 \mathrm{CP}$ media at $20 \mathrm{~d}$. Meanwhile, $71.13 \mu \mathrm{g} / \mathrm{L}$ UP concentration was detected at 2SM_2CP media after fermentation. Besides, the $\mathrm{pH}$ values in $2 \mathrm{SM} \_2 \mathrm{CP}$ and $2 \mathrm{WB} \_2 \mathrm{CP}$ media increased with the increase of time at the early fermentation stage (Fig. 4b), indicating that the nutrients of the media were utilized by the strain $S$. coelicolor. These results revealed that $\mathrm{CP}$ supplementation contributed to the increase in UP yield, and the biosynthesis of UP may be dependent on AA availability of substrates.

\subsection{Comparison of fermentation performances between $\mathrm{CP}$ and meat hydrolysates}

The hydrolysates of rabbit, beef, mutton, chicken, and duck meats were also added into MM media to compare the fermentation performances of $\mathrm{CP}$ with meat hydrolysates by S. coelicolor CGMCC 4.7172. Compared with the above plant biomass, the $\mathrm{C} / \mathrm{N}$ ratios of the chosen meat hydrolysates are relatively closer to each other, ranging from 3.54 of duck to 4.30 of rabbit (Fig. 5a). Moreover, the $\mathrm{pH}$ values in the MM media containing the five meat hydrolysates all increased with fermentation time at the early fermentation stage, and then almost remained unchanged (Fig. 5b). These facts illustrated that these meat hydrolysates can be utilized by $S$. coelicolor.

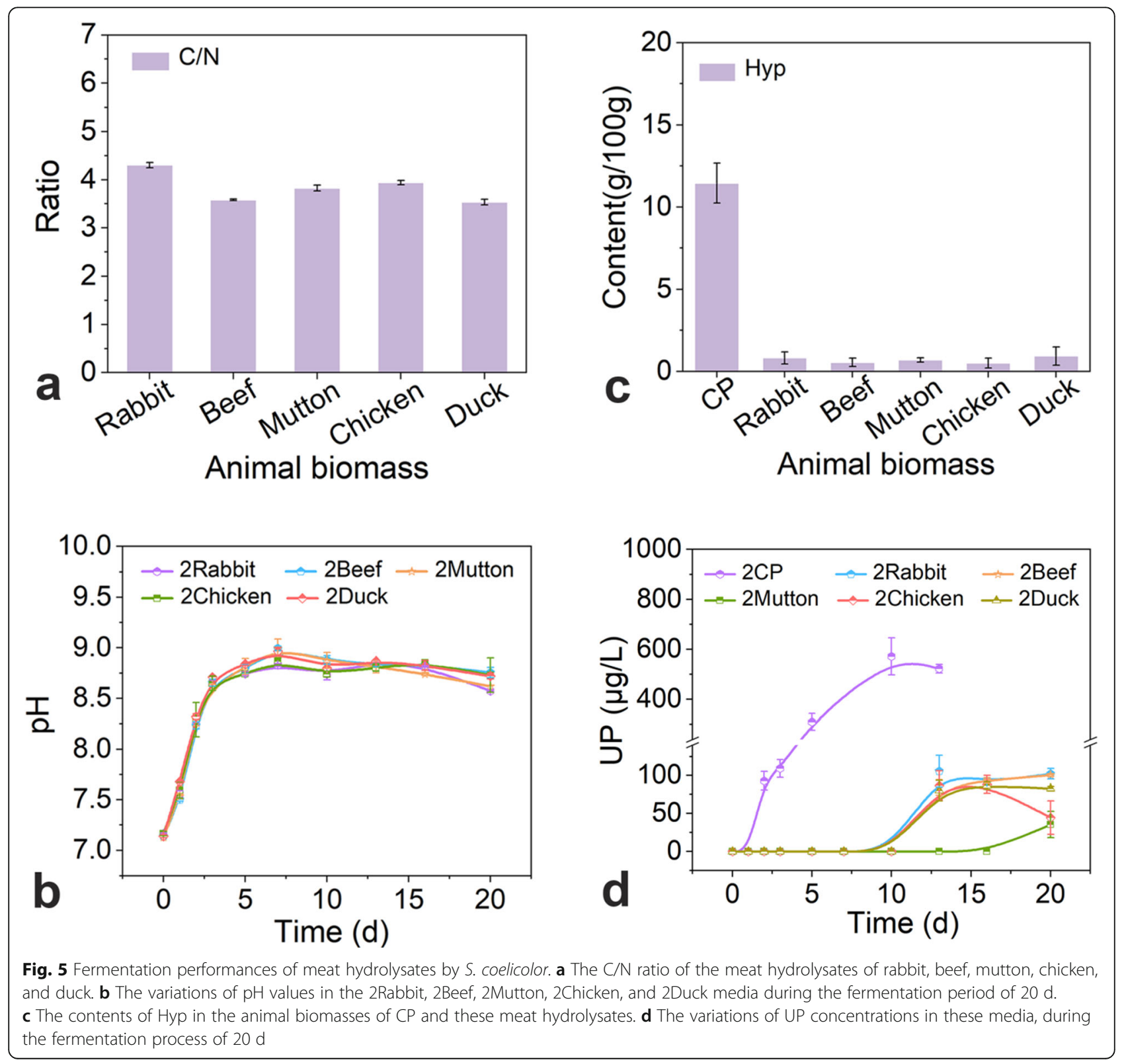


Actually, Hyp is a characteristic AA of collagen, and its content in the $\mathrm{CP}$ is up to $11.45 \mathrm{~g} / 100 \mathrm{~g}$ (Fig. 5c). Meanwhile, a small amount of Hyp was detected in the five meat hydrolysates. It was noted that the stain $S$. coelicolor cultured in the MM media containing these meat hydrolysates all produced UP during the fermentation period of $20 \mathrm{~d}$ (Fig. $5 \mathrm{~d}$ ), but the UP yields were significantly lower than that in $\mathrm{CP}$ containing media. These results illustrated that $\mathrm{CP}$ may drive the nutrient catabolism toward an increased supply of precursors, which can directly participate in UP biosynthesis.

However, UP was detected until $13 \mathrm{~d}$ in the MM media with the meat hydrolysates of rabbit, beef, chicken, and duck. Besides, the highest UP concentrations were $105.70,100.64,35.54,88.36$ and $86.83 \mu \mathrm{g} /$ $\mathrm{L}$ in the 2Rabbit, 2Beef, 2Mutton, 2Chicken, and 2Duck media, respectively. Obviously, the initial producing time was delayed and the final UP concentration was lower in these media, in comparison with that in 2CP media. Therefore, it is reasonable to believe that $\mathrm{CP}$ has a special superiority for UP production due to its unique AAs composition.

\subsection{UP producing ability of animal proteins by $S$. coelicolor}

The importance of amino acids composition of substrates to produce UP was further analyzed. BSA, casein, and keratin were selected to supplement in MM media for exploring their antibiotic producing abilities, when they acted as a sole source of carbon and nitrogen fermented by S. coelicolor CGMCC 4.7172. Actually, the C/ $\mathrm{N}$ ratios of BSA, casein, and keratin showed no obvious differences at 3.44, 3.71, and 3.15, respectively (Fig. 6a). The main chemical compositions of BSA, casein and keratin are summarized in Table S1. The content of Ser was higher, while the contents of Pro and Gly were evidently lower in BSA, casein, and keratin than those in CP (Fig. 6b- d). In particular, the Gly contents were the lowest among the three AAs for each of the animal proteins.

Fermentation experiments showed the UP was not detected in 2BSA, 2Casein, and 2Keratin media during the fermentation period of $20 \mathrm{~d}$ (Fig. 6e), possibly due to the limitation of Gly. Actually, Gly is an essential
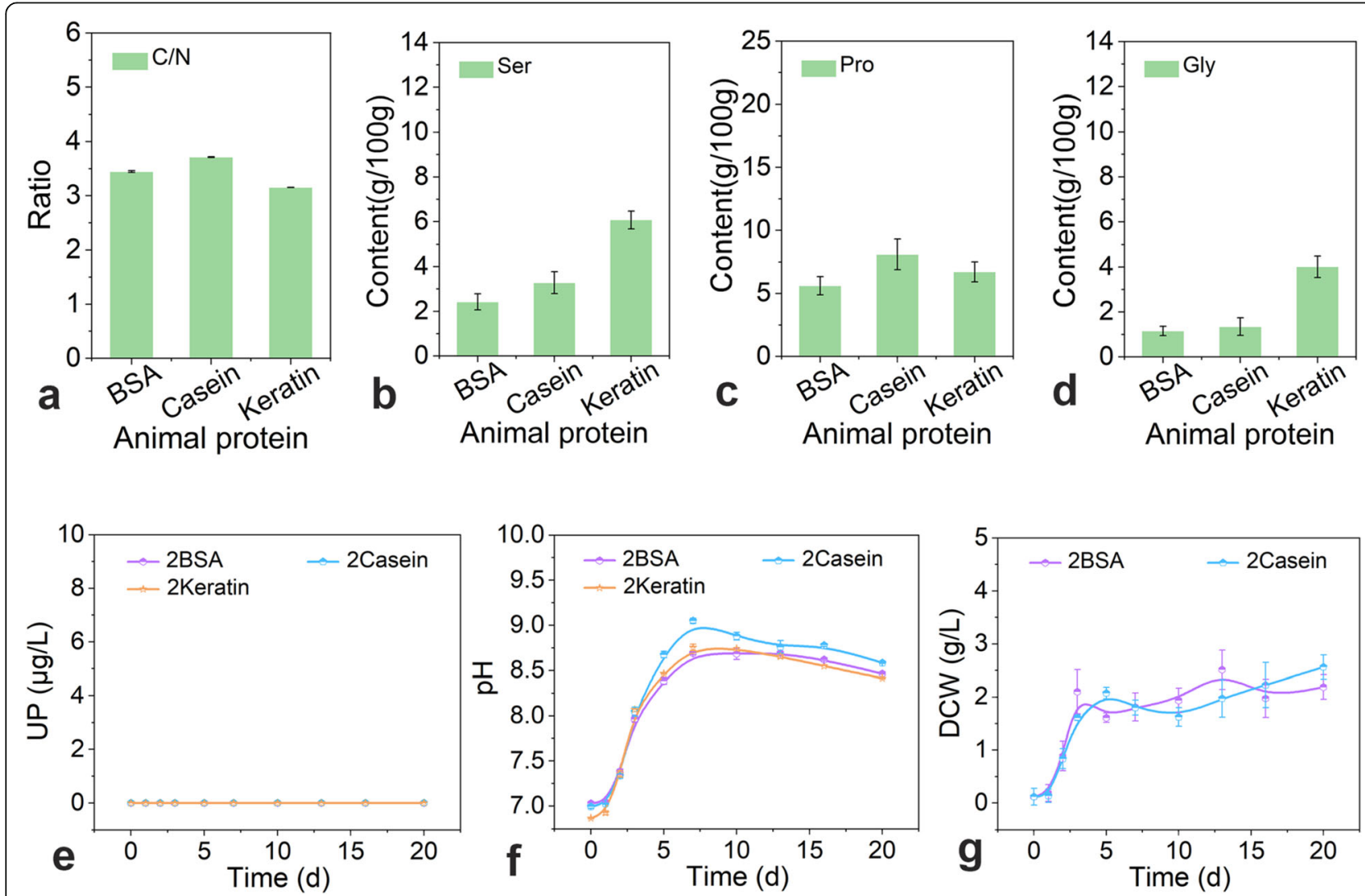

Fig. 6 The AAs characteristics and UP producing ability of the animal proteins by S. coelicolor. a The C/N ratio of the animal proteins including BSA, casein, and keratin. b-d The contents of Ser, Pro, and Gly in the three animal proteins, respectively. $\mathbf{e}$ and $\mathbf{f}$ The variations of UP concentrations and $\mathrm{pH}$ values in the 2BSA, 2Casein, and 2Keratin media, respectively, during the fermentation period of $20 \mathrm{~d}$. $\mathbf{g}$ The change of DCW in the 2BSA and 2Casein media during the fermentation process 
component to the structure of 2-undecylpyrrole, which was an intermediate for UP biosynthesis [41, 43]. In fact, the $\mathrm{pH}$ changes in these media were basically consistent with those in MM media containing meat hydrolysates (Fig. 5b and 6f). The highest $\mathrm{pH}$ values were reached at $7 \mathrm{~d}$ with 8.69, 9.05 and 8.76 in the 2BSA, 2Casein, and 2 Keratin media, respectively. In particular, the DCW increased with the prolonged time at the early fermentation stage in the 2BSA and 2Casein media (Fig. 6g), indicating the animal proteins were utilized for the growth of S. coelicolor rather than contributing to UP production. These results further demonstrated that the UP production of $S$. coelicolor is closely related to the AA composition of the substrates, and that $\mathrm{CP}$ has a high content of precursor AAs needed for UP biosynthesis.

\section{Conclusions}

This study provides novel insights regarding the application of animal biomass $\mathrm{CP}$ and the development of raw stock sources for UP production. The addition of CP can boost the growth of S. coelicolor CGMCC 4.7172. Meanwhile, CP can be used as carbon and nitrogen source for the growth and antibiotic production of S. coelicolor. Plant biomasses including SM, SP, WB and ME were unsuitable for producing UP, whereas $\mathrm{CP}$ can promote UP production. Compared with other animal biomass, $\mathrm{CP}$ is a promising biomass resource to produce UP because of its specific AA composition. In future investigations, the application behaviors of $\mathrm{CP}$ in large-scale UP production and other high value-added products by fermentation are worth anticipated.

\section{Abbreviations}

CP: Collagen peptide; UP: Undecylprodigiosin; S. coelicolor: Streptomyces coelicolor; Pro: Proline; Gly: Glycine; Ser: Serine; AAs: Amino acids; Hyp: Hydroxyproline; CGMCC: China General Microbiological Culture Collection Center; BSA: Bovine serum albumin; SM: Soybean meal; WB: Wheat bran; SP: Soybean powder; ME: Malt extract; ISP-2: International Streptomyces Project medium No.2; HPLC: High-performance liquid chromatography; C/ $\mathrm{N}$ : Carbon-to-nitrogen ratio; DCW: Dry cell weight; TOC: Total organic carbon; TNb: Total chemically bound nitrogen; MM: Minimal medium; MBC: 4methoxy-2,2'-bipyrrole-5-carbaldehyde

\section{Supplementary Information}

The online version contains supplementary material available at https://doi. org/10.1186/s42825-021-00059-y.

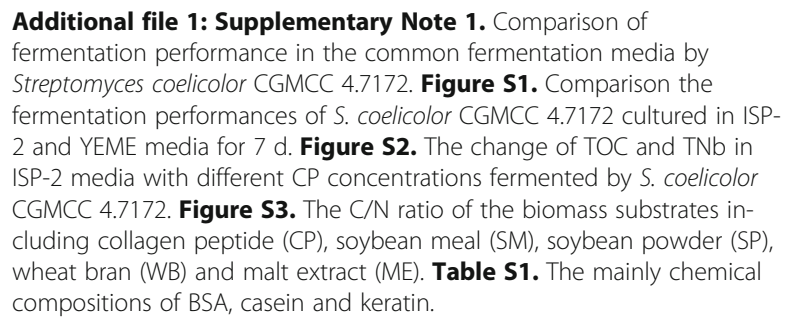

\section{Acknowledgements}

We thank Dr. Xiu He at college of biomass science and engineering, Sichuan University, for the assistance involved in the experimental equipment.

\section{Authors' contributions}

Xia Li: Conceptualization, Methodology, Investigation, Writing - original draft. Meifeng Li: Investigation, Data curation. Junling Guo: Methodology. Xian Liu: Formal analysis. Xuepin Liao: Conceptualization, Methodology, Supervision, Writing - review \& editing. Bi Shi: Conceptualization, Supervision. The author(s) read and approved the final manuscript.

\section{Funding}

We acknowledge the financial support provided by the National Key R \& D Program of China (2017YFB0308500).

\section{Availability of data and materials}

All data generated or analyzed during this study are included in this manuscript and additional file.

\section{Declaration}

Competing interests

The authors declare that they have no competing interests.

\section{Author details}

${ }^{1}$ Department of Biomass Science and Engineering, Sichuan University, 610065 Chengdu, People's Republic of China. ${ }^{2}$ National Engineering Research Center of Clean Technology in Leather Industry, Sichuan University, 610065 Chengdu, People's Republic of China. ${ }^{3}$ The Key Laboratory of Leather Chemistry and Engineering of Ministry of Education, Sichuan University, 610065 Chengdu, People's Republic of China.

Received: 26 March 2021 Accepted: 27 April 2021

Published online: 15 July 2021

\section{References}

1. Cho EJ, Trinh LTP, Song Y, Lee YG, Bae H-J. Bioconversion of biomass waste into high value chemicals. Bioresour Technol. 2020;298:122386.

2. Huang Y, Li B, Liu D, Xie X, Zhang H, Sun H, Hu X, Zhang S. Fundamental Advances in Biomass Autothermal/Oxidative Pyrolysis: A Review. ACS Sustainable Chem Eng. 2020;8(32):11888-905.

3. Chang K-H, Lou K-R, Ko C-H. Potential of bioenergy production from biomass wastes of rice paddies and forest sectors in Taiwan. J Cleaner Prod. 2019:206:460-76.

4. Isikgor FH, Becer CR. Lignocellulosic biomass: a sustainable platform for the production of bio-based chemicals and polymers. Polym Chem. 2015;6(25): 4497-559.

5. Kim SY, Yang Y-H, Choi K-Y. Bioconversion of plant hydrolysate biomass into biofuels using an engineered Bacillus subtilis and Escherichia coli mixedwhole cell biotransformation. Biotechnol Bioprocess Eng. 2020;25(3):477-84.

6. Ruiz B, Chávez A, Forero A, García-Huante Y, Romero A, Sánchez M, Rocha D, Sánchez B, Rodríguez-Sanoja R, Sánchez S. Production of microbial secondary metabolites: regulation by the carbon source. Crit Rev Microbiol. 2010;36(2):146-67.

7. Jeong Y, Kim J-N, Kim MW, Bucca G, Cho S, Yoon YJ, Kim B-G, Roe J-H, Kim SC, Smith CP. The dynamic transcriptional and translational landscape of the model antibiotic producer Streptomyces coelicolor A3 (2). Nat Commun. 2016; 7(1):1-11.

8. Nikodinovic-Runic J, Mojic M, Kang Y, Maksimovic-Ivanic D, Mijatovic S, Vasiljevic B, Stamenkovic VR, Senerovic L. Undecylprodigiosin conjugated monodisperse gold nanoparticles efficiently cause apoptosis in colon cancer cells in vitro. J Mater Chem B. 2014;2(21):3271-81.

9. Liu P, Wang Y-y, Qi X, Gu Q, Geng M, Li J. Undecylprodigiosin induced apoptosis in P388 cancer cells is associated with its binding to ribosome. PLoS One. 2013;8(6):e65381.

10. Ho T-F, Ma C-J, Lu C-H, Tsai Y-T, Wei Y-H, Chang J-S, Lai J-K, Cheuh P-J, Yeh C-T, Tang P-C. Undecylprodigiosin selectively induces apoptosis in human breast carcinoma cells independent of p53. Toxicol Appl Pharmacol. 2007; 225(3):318-28.

11. Stankovic N, Radulovic V, Petkovic M, Vuckovic I, Jadranin M, Vasiljevic B, Nikodinovic-Runic J. Streptomyces sp. JS520 produces exceptionally high 
quantities of undecylprodigiosin with antibacterial, antioxidative, and UVprotective properties. Appl Microbiol Biotechnol. 2012;96(5):1217-31.

12. Liu P, Zhu H, Zheng G, Jiang W, Lu Y. Metabolic engineering of Streptomyces coelicolor for enhanced prodigiosins (RED) production. Sci China Life Sci. 2017;60(9):948-57.

13. Palazzotto E, Tong Y, Lee SY, Weber T. Synthetic biology and metabolic engineering of actinomycetes for natural product discovery. Biotechnol Adv. 2019;37(6):107366.

14. Olano C, Lombó F, Méndez C, Salas JA. Improving production of bioactive secondary metabolites in actinomycetes by metabolic engineering. Metab Eng. 2008;10(5):281-92.

15. Darshan N, Manonmani H. Prodigiosin and its potential applications. J Food Sci Technol. 2015;52(9):5393-407.

16. Williamson NR, Fineran PC, Leeper FJ, Salmond GP. The biosynthesis and regulation of bacterial prodiginines. Nat Rev Microbiol. 2006;4(12):887-99.

17. Palazzotto E, Renzone G, Fontana P, Botta L, Scaloni A, Puglia AM, Gallo G. Tryptophan promotes morphological and physiological differentiation in Streptomyces coelicolor. Appl Microbiol Biotechnol. 2015;99(23):10177-89.

18. Maharjan S, Park JW, Yoon YJ, Lee HC, Sohng JK. Metabolic engineering of Streptomyces venezuelae for malonyl-CoA biosynthesis to enhance heterologous production of polyketides. J Biotechnol Lett. 2010;32(2):277-82.

19. Shoulders MD, Raines RT. Collagen Structure and Stability. Annu Rev Biochem. 2009:78:929-58.

20. Zhang QX, Li J, Zhang WH, Liao XP, Shi B. Adsorption chromatography separation of baicalein and baicalin using collagen fiber adsorbent. Ind Eng Chem Res. 2013;52(6):2425-33.

21. Tan Y, Chang SK. Isolation and characterization of collagen extracted from channel catfish (Ictalurus punctatus) skin. Food chem. 2018;242:147-55.

22. Ramshaw JAM, Shah NK, Brodsky B. Gly-X-Y tripeptide frequencies in collagen: A context for host-guest triple-helical peptides. J Struct Biol. 1998; 122(1-2):86-91.

23. Zhang X, Xu S, Shen L, Li G. Factors affecting thermal stability of collagen from the aspects of extraction, processing and modification. J Leather Sci Eng. 2020;2(1):19.

24. Li X, Cen N, Liu L, Chen Y, Yang X, Yu K, Guo J, Liao X, Shi B. Collagen peptide provides Saccharomyces cerevisiae with robust stress tolerance for enhanced bioethanol production. ACS Appl Mater Interfaces. 2020;12(48): 53879-90.

25. Li X, Zeng W-C, Zhu D-Y, Feng J-L, Tian C-C, Liao X-P, Shi B. Investigation of collagen hydrolysate used as carbon and nitrogen source in the fermentation of Bacillus pumilus. Process Biochem. 2017;55:11-6.

26. Thomas MG, Burkart MD, Walsh CT. Conversion of L-proline to pyrrolyl-2carboxyl-S-PCP during undecylprodigiosin and pyoluteorin biosynthesis. Chem Biol. 2002;9(2):171-84.

27. Chen $\mathrm{H}$, Wierenga PA, Hendriks WH, Jansman AJM. In vitro protein digestion kinetics of protein sources for pigs. Animal. 2019;13(6):1154-64

28. Martens BMJ, Bruininx EMAM, Gerrits WJJ, Schols HA. The importance of amylase action in the porcine stomach to starch digestion kinetics. Anim Feed Sci Technol. 2020;267:114546

29. Yin $M$, Wuyun $T$, Jiang $Z$, Zeng J. Amino acid profiles and protein quality of Siberian apricot (Prunus sibirica L.) kernels from Inner Mongolia. J For Res. 2020;31(4):1391-7.

30. Zeng W-C, Zhang W-C, Zhang W-H, Shi B. Antioxidant activity and characterization of bioactive polypeptides from bovine hair. React Funct Polym. 2013;73(3):573-8.

31. Su W-T, Tsou T-Y, Liu H-L. Response surface optimization of microbial prodigiosin production from Serratia marcescens. J Taiwan Inst Chem Eng. 2011;42(2):217-22.

32. Zöhrer $H$, Vogel F. Hydrothermal catalytic gasification of fermentation residues from a biogas plant. Biomass Bioenergy. 2013;53:138-48.

33. Simon J, Dannenmann M, Gasche R, Holst J, Mayer H, Papen H, Rennenberg H. Competition for nitrogen between adult European beech and its offspring is reduced by avoidance strategy. For Ecol Manage. 2011;262(2): 105-14

34. Lu M, Jiang Y-L, Wang S, Jin H, Zhang R-G, Virolle M-J, Chen Y, Zhou C-Z. Streptomyces coelicolor SCO4226 is a nickel binding protein. PLoS One. 2014; 9(10):e109660

35. Schäberle TF, Orland A, König GM. Enhanced production of undecylprodigiosin in Streptomyces coelicolor by co-cultivation with the corallopyronin A-producing myxobacterium, Corallococcus coralloides. Biotechnol Lett. 2014;36(3):641-8.
36. Pahira DSJ, Hertiani T, Lisdiyanti P, Ratnakomala S, Mustofa M. Isolation and antimicrobial screening of Actinomycetes from the soil of Enggano Island, Indonesia. Key Eng Mater. 2020;840:284-90.

37. Luo H, Niu Y, Duan C, Su H, Yan G. A pH control strategy for increased $\beta$ carotene production during batch fermentation by recombinant industrial wine yeast. Process Biochem. 2013;48(2):195-200.

38. Zang C-Z, Yeh C-W, Chang W-F, Lin C-C, Kan S-C, Shieh C-J, Liu Y-C. Identification and enhanced production of prodigiosin isoform pigment from Serratia marcescens N10612. J Taiwan Inst Chem Eng. 2014;45(4):1133-9.

39. Vassallo A, Palazzotto E, Renzone G, Botta L, Faddetta T, Scaloni A, Puglia AM, Gallo G. The Streptomyces coelicolor small ORF trpM stimulates growth and morphological development and exerts opposite effects on Actinorhodin and calcium-dependent antibiotic production. Front Microbiol. 2020;11:224

40. Wei YH, Yu WJ, Chen WC. Enhanced undecylprodigiosin production from Serratia marcescens SS-1 by medium formulation and amino-acid supplementation. J Biosci Bioeng. 2005;100(4):466-71.

41. Stanley $A E$, Walton $L$, Zerikly MK, Corre C, Challis GL. Elucidation of the Streptomyces coelicolor pathway to 4-methoxy-2, 2'-bipyrrole-5carboxaldehyde, an intermediate in prodiginine biosynthesis. Chem Comm. 2006;38:3981-3.

42. Wasserman HH, Shaw CK, Sykes RJ, Cushley RJ. The biosynthesis of metacycloprodigiosin and undecylprodigiosin. Tetrahedron Lett. 1974;15(33): 2787-90.

43. Mo S, Sydor PK, Corre C, Alhamadsheh MM, Stanley AE, Haynes SW, Song L, Reynolds KA, Challis GL. Elucidation of the Streptomyces coelicolor pathway to 2-undecylpyrrole, a key intermediate in undecylprodiginine and streptorubin B biosynthesis. Chem Biol. 2008;15(2):137-48.

\section{Publisher's Note}

Springer Nature remains neutral with regard to jurisdictional claims in published maps and institutional affiliations.

\section{Submit your manuscript to a SpringerOpen ${ }^{\circ}$ journal and benefit from:}

- Convenient online submission

- Rigorous peer review

- Open access: articles freely available online

High visibility within the field

- Retaining the copyright to your article

Submit your next manuscript at $\boldsymbol{\nabla}$ springeropen.com 